\title{
EXPLORACIÓN DEL RIESGO DE SUICIDIO EN ESTUDIANTES UNIVERSITARIOS MEXICANOS DURANTE EL AISLAMIENTO SOCIAL POR COVID-19
}

EXPLORING THE RISK OF SUICIDE IN MEXICAN UNIVERSITY STUDENTS

DURING SOCIAL ISOLATION BY COVID-19

Marco Antonio Santana Campas*, Laura Elena de Luna Velasco*, Evangelina Elizabeth Lozano

Montes de Oca*, Alicia Edith Hermosillo de la Torre**

Universidad de Guadalajara*, Universidad Autónoma de Aguascalientes**, México

Correspondencia:mascampas@gmail.com

\section{RESUMEN}

El aislamiento social involuntario por pandemia tiene consecuencias en todos los ámbitos sociales. El aislamiento social conlleva reacciones emocionales, comportamentales y psicológicas que pueden llevar al suicidio cuando las personas se sienten rebasadas y las expectativas sobre el futuro son catastróficas. Objetivo. Describir los estresores, cambio de rol, reacciones emocionales y riesgo de suicidio ante el aislamiento social por COVID-19 en estudiantes universitarios. Material y Método: Estudio cualitativo, descriptivo, exploratorio y transversal, con un muestreo por conveniencia. Participaron 1,186 estudiantes universitarios, la media de edad fue de 20 y DE 3.5, estado civil $94.8 \%$ solteros y $3.8 \%$ reportaron estar en una relación (casados o concubinato) y el 3.5\% refirió que vive solo, $66.3 \%$ mujeres $(n=786)$ y $33.7 \%$ hombres $(n=400)$. Instrumento: cuestionario exprofeso para identificar variables sociodemográficas, 
emocionales, psicológicas, cambios de rol, consumo de alcohol y tabaco, prácticas de autocuidado para inferir para el riesgo de suicidio. Resultados: el $32.6 \%$ de los encuestados reportó que no tiene un plan de actividades que como mínimo incluya: trabajo académico, trabajo en casa, esparcimiento, hora para dormir y despertar. Se identificó que el 3.5 y el 6.4\% aumentó el consumo de tabaco y alcohol respectivamente. El 47.5\% refirió que al estar nuevamente con sus familias no se sienten integrados, se sienten frustrados, estresados, aburridos y como si no estuvieran en casa. Las emociones y sentimientos más frecuentes son: miedo, enojo, sensación de pérdida de control, tristeza, desorganización, sensación de encierro, aburrimiento, frustración y ansiedad, esto puede desencadenar ideación y conductas suicidas.

Palabras clave: Suicidio, aislamiento social, estudiantes, coronavirus.

\section{ABSTRACT}

Involuntary social isolation due to a pandemic has consequences in all social areas. Social isolation involves emotional, behavioral, and psychological reactions that can lead to suicide when people feel overwhelmed and expectations about the future are catastrophic. Objective. Describe stressors, role change, emotional reactions, and suicide risk in the face of social isolation by COVID-19 in university students. Material and Method: Qualitative, descriptive, exploratory and cross-sectional study, with convenience sampling. 1,186 university students participated, the average age was 20 and SD 3.5, marital status 94.8\% single and 3.8\% reported being in a relationship (married or in concubinage) and 3.5\% reported that they live alone, $66.3 \%$ women $(n=786)$ and $33.7 \%$ men $(n$ =400). Instrument: express questionnaire to identify sociodemographic, emotional, psychological variables, role changes, alcohol and tobacco 
consumption, self-care practices. To infer for suicide risk. Results: $32.6 \%$ of the respondents reported that they do not have an activity plan that at least includes: academic work, work at home, recreation, time to sleep and wake up. It was identified that 3.5 and $6.4 \%$ increased tobacco and alcohol consumption respectively. $47.5 \%$ reported that when they were back with their families they did not feel integrated, they felt frustrated, stressed, bored and as if they were not at home. The most recurring emotions and feelings are: fear, anger, feeling of loss of control, sadness, disorganization, feeling of being closed, boredom, frustration and anxiety, this can trigger suicidal ideation and behaviors.

Key words: Suicide, social isolation, students, coronavirus.

\section{INTRODUCCIÓN}

El denominado COVID-19 ha sorprendido al mundo, según el último reporte de la Organización Mundial de la Salud (OMS) al 17 de mayo del 2020 existían 4,534,731 personas infectadas y 307,537 defunciones a causa de este virus. Ante la falta de vacunas y medicamentos para tratar esta enfermedad, ha llevado a los gobiernos a implementar las clásicas estrategias de salud pública como el distanciamiento social, aislamiento, cuarentena y contención comunitaria, con el propósito de prevenir la propagación de la enfermedad de persona a persona separando a las personas para interrumpir la transmisión (OMS, 2020; Wilder-Smith \& Freedman 2020).

Sin duda, las estrategias de salud pública disponibles están dando resultado para controlar el contagio del COVID-19, pero ahora el reto eminente son las consecuencias económicas, políticas, sociales y salud mental a corto plazo. Además, para el cumplimiento de las medidas de salud pública se 
involucra las consecuencias económicas, aumento sin precedente en el desempleo y afectaciones psicológicas, la confianza en las autoridades que las emiten, la duración y claridad con las que son pronunciadas.

El aislamiento social y la limitación de movilidad sin fecha de finalización, aumenta la gravedad percibida del motivo de aislamiento, lo que podría aumentar el incumplimiento, además, esto puede aumentar la ansiedad y otras afectaciones psicológicas por la incertidumbre, de esta manera, esta enfermedad infecciosa no solo afecta la salud física de los pacientes, sino que también afectan la salud psicológica y el bienestar de la población no infectada (Briscese, Lacetera, Macis \& Tonin, 2020; Giselly-Mayerly \& Nieves-Cuervo, 2020; Han, Yan, Desheng, Shiyue, Ningxi, 2020; Weems, Carrion, McCurdy, Scozzafava, 2020).

Dentro de las afectaciones psicológicas del aislamiento social está la ideación y conductas suicidas. El sentimiento subjetivo de soledad tiene un gran impacto en la ideación suicida, por tanto, se debe vigilar el aislamiento social objetivo y subjetivo para identificar el riesgo de suicidio. Las principales construcciones sociales asociadas con los resultados suicidas son el estado civil (soltero, separado, divorciado o viudo), vivir solo, aislamiento social, soledad, alienación, sentido de pertenencia, desconsuelo, impotencia, desesperanza, estrés y angustia (Giselly-Mayerly \& Nieves-Cuervo, 2020; Calati, Ferrari, Brittner, Oasi, Olié, Carvalho, Courtet, 2019).

Otras de las consecuencias psicológicas por el aislamiento social asociadas al riesgo de suicidio son: la tristeza, la preocupación, el miedo, la ira, la molestia, la frustración, la culpa, la impotencia, la soledad y el nerviosismo (Calati, Ferrari, Brittner, Oasi, Olié, Carvalho, Courtet, 2019; Dueweke, 
Schwartz-Mette, 2018). En Estados Unidos de Norteamérica se realizó un estudio para identificar las afectaciones del aislamiento y su relación con la ideación suicida en el último mes, los resultados apoyan una asociación entre varias experiencias relacionadas con COVID-19 (es decir, angustia general, miedo al daño físico, efectos de las políticas de distanciamiento social) y las ideas e intentos suicidas en el último mes.

Además, las afectaciones psicológicas por el aislamiento social, se están afectado la calidad del sueño, potencializando o agravando las afectaciones emocionales y los comportamientos desadaptativos, y este último puede ser un mediador de síntomas depresivos y riesgo de suicidio (Han, Yan, Desheng, Shiyue, Ningxi, 2020; Weems, Carrion, McCurdy, Scozzafava, 2020).

Debido a lo anterior se pronostica un aumento de la tasa de suicidios en todo el mundo debido al aumento del desempleo y el aislamiento social podría estar cerca de 50,000 personas según las estimaciones iniciales de estos impactos colaterales (Weems, Carrión, McCurdy \& Scozzafava, 2020) de hecho ya se han reportados casos de suicidio en India, Bangladesh e Italia, donde no solo han sido víctimas de población general, sino también trabajadores de la salud debido a el miedo a la infección, la exposición de los miembros de la familia, los colegas enfermos, la escasez de equipos de protección personal necesarios, las instalaciones abrumadas y el estrés laboral, por ejemplo, en Italia dos enfermeras infectadas se suicidaron (Mamun \& Griffiths, 2020; Reger, Stanley, Joiner; 2020; Montemurro, 2020).

La pandemia de COVID-19 no solo ha causado desafíos importantes para el sistema de salud en todo el mundo, sino que también ha alimentado el 
aumento de numerosos rumores, engaños y desinformación, con respecto a la etiología, los resultados, la prevención y la cura de la enfermedad, aumentando con esto las reacciones emocionales (Tasnim, Hossain \& Mazumder, 2020).

Ahora bien, en "condiciones normales o cotidianas" los estudiantes universitarios son una población vulnerable para diferentes afectaciones como, el consumo de sustancias, estrés académico, depresión, ansiedad, aislamiento social, riesgo de suicidio y suicidio, se ha encontrado evidencia que el sexo (sexo femenino es el más afectado en el intento y en el suicidio, el masculino), edad, antecedentes de autolesión, estado civil (solteros, vivir solo, divorciados), desesperanza, impulsividad y trastorno límite son predictores del suicidio en estudiantes universitarios y el lugar más recurrente para consumar la conducta suicida es el hogar (González, Medina \& Ortiz 2016; Corona, Sagué, Cuellar, Hernández \& Larín, 2017; Medina, Ospina \& Cardona, 2017; Forero, Siabato \& Salamanca, 2017). La evidencia muestra que la depresión, funcionamiento familiar, pueden ser mediadores entre el consumo de sustancias y el estrés académico y la ideación suicida (Restrepo, Sánchez, Calderon, Castañeda, Osorio \& Diez, 2018).

El aislamiento social trabajo consigo la reconfiguración de roles y vínculos, entendiendo estos como la estructura de las relaciones y los grupos, en este sentido, para Podcamisky, (2006) el rol puede entenderse como "un modelo organizado de conductas, relativo a una cierta posición del individuo en una red de interacción, ligado a expectativas propias y de los otros" (p.181), el aislamiento social y confinamiento llevó a la modificación, reestructuración o regreso a roles pasados dejando vínculos actuales para reencontrarse con otros, por ejemplo, la educación universitaria supone una 
movilidad nacional o internacional en los jóvenes para cursar un pregrado o posgrado, esto supone dejar y adquirir nuevos roles y vínculos, y como ya se ha a mencionado el aislamiento social y confinamiento llevó a los estudiantes a regresar su lugar de origen, dejando las estructuras sociales, roles y vínculos establecidos con sus pares, pudiendo ser esto un fuente de estrés y otras afectaciones emocionales.

El cambio de rol supone una fuente de estrés, entendiendo este último como resultado de la interacción del individuo con su medio ambiente $\mathrm{y}$ que en situaciones que él mismo, se valora rebasado lleva a reacciones emocionales, conductuales, cognitivas y fisiológicas, creando alteraciones cuando la exposición es prolongada, en estudiantes universitarios, las principales reacciones ante el estrés son: ansiedad, afecta el rendimiento académico, puede fomentar el consumo de drogas, alteración del sueño, cambio en los hábitos alimenticios y evitación de la responsabilidad. En estudiantes universitarios, además del cambio de rol los principales generadores de estrés son la sobrecarga de tareas y trabajos escolares, falta de planeación (por parte de los docentes) y falta de estrategias de afrontamiento ante el estrés, aislamiento social y confinamiento (Águila, Calcines, Monteagudo y Nieves, 2015; Pascoe, 2020; Otero-Marrugo, 2020; Wen-Jun, 2020).

Dentro de los factores protectores para disminuir las afectaciones psicológicas por el aislamiento social por COVID-19, está el aumento del capital social, es decir, mantenerse en contacto con familias, amigos, compañeros de trabajo y de escuela, esto mejora la calidad del sueño y ayuda a reducir la ansiedad y el estrés, por tanto, además del capital social, se deberá, potenciar las emociones positivas. Además, enfocar intervención para el trabajo con el área afectiva (miedo, ansiedad, 
depresión), comportamental (conductas salutogénico, autocuidado, inmovilidad, evitación y aproximación) y el área cognitiva (desesperanza, amenaza, perdidas, frustración) (Pakpour \& Griffiths, 2020; Martínez, 2020; Tizón, 2020).

\section{Dado lo anterior, esta investigación se planteó como objetivo:}

Describir los estresores, cambio de rol, reacciones emocionales y riesgo de suicidio ante el aislamiento social por COVID-19 en estudiantes universitarios.

\section{MATERIAL Y MÉTODOS}

Estudio cualitativo, exploratorio y transversal, con un muestreo por conveniencia. Participaron 1,186 estudiantes del Centro Universitario del Sur de la Universidad de Guadalajara de 23 disciplinas (medicina, psicología, abogado, trabajo social, ingenierías, por mencionar algunas).

Se diseño un instrumento exprofeso con preguntas abiertas y cerradas para identificar variables sociodemográficas, emocionales, psicológicas, cambios de rol, consumo de alcohol y tabaco, prácticas de autocuidado. Para con esto inferir si los participantes presentaban reacciones propias para el riesgo de suicidio.

Debido a que la Universidad de Guadalajara y las Universidades públicas y privadas de México migraron al $100 \%$ de las clases a la modalidad virtual desde el 17 de marzo del 2020, el instrumento se creó en Google forms y fue distribuido por correo electrónico y WhatsApp por los coordinadores de cada carrera y por los investigadores.

Los análisis para las preguntas cerradas se realizaron con descriptivos y frecuencias, para las preguntas abiertas se realizó análisis de contenido. 
Para los análisis estadísticos se utilizó con el paquete Statistical Package for the Social Sciences versión 24 (SPSS Inc., Chicago, IL, USA). El análisis de contenido se utilizó la estrategia de categorización, estructuración, contrastación y teorización (Martínez, 2006)

Está investigación tuvo el apoyo y aval por las autoridades del Centro Universitario del Sur de la Universidad de Guadalajara en Jalisco México. Dentro del cuestionario se informó a los participantes de la naturaleza del estudio y en todo momento conservó la libertad de participar o no en la investigación, además se cuido la confidencialidad, no maleficencia y respeto a los derechos humanos.

\section{RESULTADOS}

Participaron 1,186 estudiantes universitarios, la media de edad fue de $20 \mathrm{y}$ $D E 3.5$, el estado civil predominante fue $94.8 \%$ solteros y $3.8 \%$ reportaron estar en una relación (casados o concubinato) y el 3.5\% refirió que vive solo, $66.3 \%$ mujeres $(n=786)$ y $33.7 \%$ hombres $(n=400)$. En cuanto a la ocupación el $39.1 \%$ refirió tener un empleo y estudiante, el $60.9 \%$ reportó ser estudiantes de tiempo completo.

En cuanto al uso de sustancias, en este estudio solo se identificó el consumo de alcohol y tabaco, de los encuestados el $14.1 \%$ reportó que consumía tabaco, de este porcentaje el 3.5\% reportó que durante el aislamiento social a aumentado el consumo de tabaco, en cuanto al alcohol, el 52.4 reportó consumo y durante el aislamiento el 6.4\% aumentó el consumo de alcohol.

Como ya se mencionó, desde el 17 de marzo las universidades migraron a clases virtuales durante el aislamiento social, entonces, el aislamiento 
social y las actividades virtuales generaron un sin número de estresores en los estudiantes, mismos que van desde la frustración por no tener equipo de cómputo o acceso de internet ilimitado para permanecer en contacto con compañeros y profesores, a esto se sumó la falta de dominio de las plataformas virtuales, esto lo reportó el $61.8 \%$ de los estudiantes encuestados.

El Centro Universitario del Sur se encuentra en el Municipio de Zapotlán el Grande Jalisco y de la muestra de estudio solo el 38.4\% de los estudiantes radica en este municipio, lo que implicó que durante el aislamiento social y las clases en línea la mayoría de los estudiantes regresaron a sus lugares de origen con sus familias, en cuanto a esto último, el 91.8\% de los estudiantes refirió que aún vive con su familia primaria (padres, hermanos y algún otro familiar), al regresar e integrarse con sus familias el 52.5\% reportó sentirse útil, satisfecho y responsable con su familia, pero el 47.5\% refirió que al estar nuevamente de tiempo completo con sus familias no se sienten integrados, se sienten frustrados, estresados, aburridos, como si no estuvieran en casa y con deseos de regresar a la universidad de manera física. Asimismo, al regresar a casa, además del trabajo académico, los estudiantes se involucraron en el cuidado de los hermanos menores y ayudarles en sus tareas, cuidado de familiares enfermos y en el trabajo doméstico cotidiano.

En el cuestionario se incluyeron preguntas abiertas para que de manera libre expresaran sus emociones, pensamientos y actitudes frente al aislamiento social, trabajo virtual y al reintegrarse a sus familias. Las narrativas fueron categorizadas y estructuraron, en la tabla 1 se describen los resultados de este proceso. 


\begin{tabular}{|c|c|c|c|}
\hline Número de veces & Contenido de narrativa & Número de veces & Contenido de narrativa \\
\hline 85 & Ambivalencia & 386 & Tristeza \\
\hline 124 & Rebasado & 429 & Desorganización \\
\hline 183 & Pérdida de control & 502 & Encierro \\
\hline 218 & Soledad & 593 & Aburrimiento \\
\hline 309 & Enojo & 628 & Frustración \\
\hline 368 & Miedo & 601 & Ansiedad \\
\hline
\end{tabular}

De los resultados cualitativos se resalta que solo de cuatro narrativas su contenido fue referente a estados de tranquilidad, del resto de las narrativas se puede inferir que los encuestados están en estados de ansiedad, estrés, tristeza y desesperanza.

En cuanto a las prácticas de autocuidado y hábitos de sueño, el 32.6\% reportó que no tiene un plan de actividades donde, como mínimo incluya: trabajo académico, trabajo en casa, esparcimiento, hora para dormir y despertar, el $67.4 \%$ reportó que, si realizo un plan de actividades con esos mínimos, pero solo el $41.8 \%$ cumple dicho plan de actividades diarias. En cuanto a los hábitos de sueño, el $42.8 \%$ reportó que duerme menos de ocho horas durante la noche, solo el 13\% reportó tener y respetar un horario para dormir y para despertar.

\section{DISCUSIÓN}

Al dictarse la medida de distanciamiento social y posteriormente el aislamiento social y el confinamiento, las universidades cambiaron las clases presenciales a la modalidad virtual, esto también implicó cambios de rol y las reacciones emocionales y afectaciones a la salud mental, al percibir esto en los estudiantes universitarios fue que se planteó como 
objetivo: describir los estresores, cambio de rol, reacciones emocionales y riesgo de suicidio ante el aislamiento social por COVID-19 en estudiantes universitarios, para con esto tener un panorama general desde los estudiantes sobre las afectaciones y prácticas de autocuidado. Una vez que se describieron los resultados cualitativos y cuantitativos, se procede a la contrastación y teorización y establecer la relación entre las principales reacciones de los estudiantes con un posible riesgo de suicidio en función de la literatura (Martínez, 2006).

La caracterización sociodemográfica permite identificar que los estudiantes están en algunas de las condiciones para inferir el riesgo de suicidio a nivel de ideación: vivir solo, solteros, estrés cambios en la calidad del sueño, tristeza, desorganización, encierro, aburrimiento, frustración, ansiedad, cambio súbito de rol, entre otras, por tanto, con fundamento en la literatura y la respuestas de los estudiantes, se considera que la muestra se encuentra en riesgo de ideación de suicidio, por lo que se considera de interés el seguir estudiando las reacciones emocionales frente al aislamiento social a los estudiantes universitarios para poder precisar el porcentaje que cumplen criterios específicos para el riesgo de suicidio, para así tener un acercamiento a la realidad y poder implementar programas preventivos (Giselly-Mayerly \& Nieves-Cuervo, 2020; Calati, Ferrari, Brittner, Oasi, Olié, Carvalho \& Courtet, 2019; Dueweke \& Schwartz-Mette, 2018).

Asimismo, se identificó que el 3.5 y el $6.4 \%$ aumentó el consumo de tabaco y alcohol respectivamente, el $32.6 \%$ reportó que no tiene un plan de actividades donde, como mínimo incluya: trabajo académico, trabajo en casa, esparcimiento, hora para dormir y despertar, esto se puede considerar como comportamientos desadaptativos y no salutogénicos mismos que pueden potenciar las afectaciones emocionales y síntomas depresivos y a 
su vez a conductas o ideación suicida (Han, Yan, Desheng, Shiyue, Ningxi, 2020; Weems, Carrion, McCurdy, Scozzafava, 2020; Dueweke, SchwartzMette, 2018; Reger, Stanley \& Joiner, 2019; Montemurro, 2020).

Las prácticas de autocuidado son todas aquellas acciones encaminadas a mejorar la calidad de vida de manera general con la finalidad de mantener en equilibrio y la funcionalidad de la persona en todas sus dimensiones. En la muestra de estudio se identificó que el 67.4\% realizó un plan de actividades durante el aislamiento social, pero solo el $41.8 \%$ cumple dicho plan, aumentando con esto la sensación de falta de control y sentirse rebasados, por consiguiente, esto se convierte en potencializador para la frustración, ansiedad, ira e impotencia, siendo estos factores para el riesgo de suicidio (Calati, Ferrari, Brittner, Oasi, Olié, Carvalho, Courtet, 2028; Dueweke, Schwartz-Mette, 2028; Reger, Stanley \& Joiner, 2019). Pero el hecho de realizar un plan de actividades, también se puede interpretar como el esfuerzo que se está realizando para hacer frente a las actividades académicas, de trabajo y de reintegrarse a la familia, por tanto, se considera pertinente brindar apoyo para que estos esfuerzos no se vean frustrados y por consiguiente sea un generador de estrés.

\section{CONCLUSIONES}

En condiciones "normales y cotidianas" es decir sin la pandemia por COVID-19, el suicidio es la segunda causa de muerte en el grupo etario de 15 a 29 años, rango de edad en la que se encuentran los estudiantes universitarios, ahora, con las reacciones del aislamiento social puede incrementarse la incidencia del suicidio durante y posterior al aislamiento social (Weems, Carrión, McCurdy \& Scozzafava, 2020). 
Derivado de lo anterior, se concluye que, tratándose de estudiantes universitarios, las instituciones educativas tienen la responsabilidad social en la formación del alumnado, y por consiguiente debe potencializar que los estudiantes realicen un plan de actividades, mantener el contacto con la institución y con sus pares, para con esto favorecer el mantenimiento e incremento del capital social en los jóvenes y sus familias. Ante esto pueden ser estrategias los webinar para brindar estrategias de afrontamiento al estrés, autocuidado de la salud física, psicológica y hábitos de estudio, grupos para compartir y escucha haciendo uso de las plataformas virtuales con actividades sincrónicas y asincrónicas, donde el contenido pueden ser reflexiones, explicación y modelado de diferentes estrategias para la regulación emocional y actividades de autocuidado.

Es responsabilidad de los Gobiernos y las Universidades ampliar los servicios de salud y promoción de prácticas de autocuidado para disminuir las consecuencias psicológicas de la población general, pero con especial atención a los grupos vulnerables, la poca literatura existente sobre el tema particular empieza a evidenciar algunas estrategias para disminuir las afectaciones por el aislamiento social, a continuación, se mencionan algunas:

I Psicoeducar para resolución de conflictos personales e interpersonales, esto a través de talleres virtuales con actividades sincrónicas y asincrónicas.

I Facilitar, por los medios disponibles el acompañamiento a los estudiantes universitarios para la expresión de dificultades académicas, emocionales y comportamentales.

I Desarrollar programa de formación, capacitación y actualización permanente dirigido a los profesores y especialistas que brindan la atención en crisis bajo modelos de atención psicológica basados en evidencia para la atención psicológica en el fenómeno suicida. 
I Promover en el trabajo docente vínculos académicos que promuevan y desarrollen recursos de afrontamiento en la relación maestroalumno.

I Una vez concluya el aislamiento, priorizar en las Universidades el ofrecer el apoyo psicológico que se requiera para los casos identificados en riesgo de suicidio, brindando los apoyos individuales, grupales y seguimiento interinstitucional para asegurar su atención.

\section{LIMITANTES}

Se recomienda que en investigaciones futuras se utilice una escala de riesgo de suicidio para que los resultados de esta puedan ser triangulada con los datos cualitativos y con esto poder realizar modelos predictivos.

\section{AGRADECIMIENTOS}

Se agradece a las autoridades del Centro Universitario del Sur de la Universidad de Guadalajara por autorizar y apoyar la realización de esta investigación, con su apoyo se logro el objetivo de tener el aval de la institución para que fuera difundido y aplicado en los distintos Programas Educativos que se ofertan en el Centro Universitario del SUR de la Universidad de Guadalajara, logrando tener la representatividad de una muestra solida de estudiantes.

\section{REFERENCIAS}

I Águila, B., Calcines M., Monteagudo, R. \& Nieves, Z. (2015). Estrés académico. Revisión. EDUMECENTRO V.7(2):163-178. Recuperado de: https://www.medigraphic.com/pdfs/edumecentro/ed2015/ed152m.pdf

I Briscese G, Lacetera N, Macis M, \& Tonin M. (2020) Compliance with covid-19 social-distancing measures in italy: the role of expectations and duration. NBER Working Paper No. 26916:1-27. Recuperado de https://www.nber.org/papers/w26916 
I Calati, R., Ferrari, Ch., Brittner, M., Oasi, O., Olié, E., Carvalho, A. \& Courtet, P. (2019). Suicidal thoughts and behaviors and social Isolation: a narrative review of the literature. Journal of Affective Disorders. V. 245: 653-667. https://doi.org/10.1016/j.jad.2018.11.022

I Corona, B., Sagué, K., Cuellar, L., Hernández, M. \& Larín, S. (2017). Caracterización de la Conducta suicida en Cuba, 2011-2014. Revista Habanera de Ciencias Médicas; 16(4): 612-624. Recuperado de http://scielo.sld.cu/scielo.php?script=sci_arttext\&pid=S1729-519X2017000400013

I Dueweke, A. \& Schwartz-Mette, R. (2018). Social-Cognitive and Social Behavioral Correlates of Suicide Risk in College Students: Contributions from Interpersonal Theories of Suicide and Depression. Archives of Suicide Research. 22:224-240. DOI: 10.1080/13811118.2017.1319310

I Forero, I., Siabato, E. \& Salamanca, Y. (2017). Ideación suicida, funcionamiento familiar y consumo de alcohol en adolescentes colombianos. Revista Latinoamericana de Ciencias Sociales, Niñez y Juventud, 15(1): 431-442. Recuperado de http://www.scielo.org.co/scielo.php?pid=S1692715X2017000100028\&script=sci_abstract\&tlng=es

1 Giselly-Mayerly \& Nieves-Cuervo (2020). Covid, más allá de una pandemia. Revista Salud UIS.52(2): 175-176. Recuperado de https://revistas.uis.edu.co/index.php/revistasaluduis/article/view/10733/10500

I González, P., Medina, O. \& Ortiz, J. (2016). Riesgo suicida y factores asociados en estudiantes de Psicología en una Universidad pública de Colombia. Revista Habanera de Ciencias Médicas, 15(1):136146. Recuperado de http://scielo.sld.cu/scielo.php?script=sci_arttext\&pid=S1729-519X2016000100015

I Han, X., Yan, Z., Desheng, K., Shiyue L. \& Ningxi Y. (2020). Social Capital and Sleep Quality in Individuals Who Self-Isolated for 14 Days During the Coronavirus Disease 2019 (COVID-19). Medical science monitor: international medical journal of experimental and clinical research, 26, e923921. https:// doi.org/10.12659/MSM.923921

I Mamun, M. \& Griffiths, M. (2020). First COVID-19 suicide case in Bangladesh due to fear of COVID-19 and xenophobia: Possible suicide prevention strategies. Asian J Psychiatr, 51: 102073. DOI: 10.1016/j. ajp.2020.102073

I Martínez, C. (2020). Intervención y psicoterapia en crisis en tiempos del coronavirus (para psicólogas/os clínicos y psicoterapeutas). Centro de estudios en psicología clínica y psicoterapia. Facultad de psicología. Instituto Milenio para la Investigación en Depresión y Personalidad. Santiago, Abril 2020

I Martínez, M. (2006). Ciencia y arte en la investigación cualitativa. Segunda Edición, México. Editorial Trillas. 2006

I Medina, O.A., Ospina, S.M. \& Cardona, D.V. (2017). Caracterización del suicidio en adolescentes del Departamento de Quindío. Colombia, 1989-2013. Revista Habanera de Ciencias Médicas; 16(5): 784-795. Recuperado de http://scielo.sld.cu/scielo.php?script=sci_arttext\&pid=S1729-519X2017000500011

I Montemurro, N. (2020). The emotional impact of COVID-19: From medical staff to common people. Brain Behav Immun: 2-3 doi: 10.1016/j.bbi.2020.03.032

I Organización mundial de la salud (2020). WHO Coronavirus Disease (COVID-19) Dashboard. Recuperado de: https://covid19.who.int/ (consultado el 15 de abril del 2020) 
I Otero-Marrugo, G., Carriazo-Sampayo, G., Tamara-Oliver, S., Lacayo-Lepesqueur, MK., Torres-Barrios, G., Pájaro-Castro, N. (2020). Nivel de estrés académico por evaluación oral y escrita en estudiantes de Medicina de una universidad del Departamento de Sucre. Revista CES Medicina 2020; 34(1): 40-52. Recuperado de: http://revistas.ces.edu.co/index.php/medicina/article/view/5229

I Pakpour, A.H. \& Griffiths, M.D. (2020). The fear of COVID-19 and its role in preventive behaviors. Journal of Concurrent Disorders: Vol. (TBD) No. (TBD), (pp. TBD). http://irep.ntu.ac.uk/id/eprint/39561/

I Pascoe, M., Hetrick, S. \& Parker, A. (2020) The impact of stress on students in secondary school and higher education, International Journal of Adolescence and Youth, 25:1, 104-112, DOI: $10.1080 / 02673843.2019 .1596823$

I Podcamisky, M. (2006). El rol desde una perspectiva vincular. Reflexiones, V. 85, (1-2) pp. 179-187. Recuperado de: https://www.redalyc.org/pdf/729/72920817012.pdf

I Reger, M., Stanley, I. \& Joiner, T. (2020). Suicide Mortality and Coronavirus Disease 2019-A Perfect Storm?. JAMA Psychiatry. 2020: 1-2 doi:10.1001/jamapsychiatry.2020.1060

I Restrepo, J., Sánchez, O., Calderon, G., Castañeda, T., Osorio, Y. \& Diez, P. (2018). Depresión y su relación con el consumo de sustancias psicoactivas, el estrés académico y la ideación suicida en estudiantes universitarios colombianos. Health and Addictions; 18(2): 227-239. Recuperado de https://dialnet.unirioja. es/servlet/articulo?codigo $=6546342$

I Tasnim, S., Hossain, M. \& Mazumder, H. (2020). Impact of rumors or misinformation on coronavirus disease (COVID-19) in social media. SocArXiv. 1-8 https://doi.org/10.31235/osf.io/uf3zn

I Tizón. J. (2020). Salud emocional en tiempos de pandemia. Reflexiones urgentes. Herder Editorial, S.L., Barcelona, España. 1. ${ }^{a}$ edición digital, 2020

I Weems, C., Carrion, V., McCurdy, B. \& Scozzafava, M. (2020). Increased Risk of Suicide Due to Economic and Social Impacts of Social Distancing Measures to Address the Covid-19 Pandemic: A Forecast. Source: Researchgate.net. 1-10 DOI: 10.13140/RG.2.2.21601.45926

I Wen-Jun Z., Chao Y., Shumb, D. \& Ci-Ping D. (2020). Responses to academic stress mediate the association between sleep difficulties and depressive/anxiety symptoms in Chinese adolescents. Journal of Affective Disorders. V. 263, 89-98. https://doi.org/10.1016/j.jad.2019.11.157

$\checkmark$ Wilder-Smith A. \& Freedman D. (2020). Isolation, quarantine, social distancing and community containment: pivotal role for old-style public health measures in the novel coronavirus (2019-nCoV) outbreak. Journal of Travel Medicine;27(2): 1-4. DOI: 10.1093/jtm/taaa020

\section{Envío a dictamen: 30 de mayo de 2020 Reenvío: 18 de junio de 2020 Aprobación: 3 de julio de 2020}


Marco Antonio Santana Campas. Doctor en psicología con orientación en calidad de vida y salud. Docente de pregrado y posgrado en el Centro Universitario del Sur de la Universidad de Guadalajara y en el ITESO: Universidad Jesuita de Guadalajara. Miembro del Sistema Nacional de Investigadores del CONACyT. Ha coordinado libros y ha publicado diversos capítulos en libros arbitrados y artículos en revistas internacionales con temas sobre las violencias, tratamiento penitenciario, suicidio, drogas y habilidades para la vida. Correo electrónico: mascampas@gmail.com

Laura Elena De Luna Velasco. Maestría en Metodología de la Enseñanza. Cuenta con reconocimiento al perfil deseable PRODEP. Trabaja las líneas de investigación en innovación, tecnología educativa y desarrollo humano, inclusión y riesgos psicosociales. Línea de investigación relacionada con la identificación de factores de riesgo en estudiantes universitarios, barreras en el aprendizaje y la participación, percepciones sobre discapacidad de estudiantes universitarios desde la mirada de alumnos(as) y estudiantes con alguna condición permanente o transitoria de discapacidad. Correo electrónico: laura.luna@cusur.udg.mx

Evangelina Elizabeth Lozano Montes de Oca. Doctora en Derecho, profesora de tiempo completo y Jefa del Departamento de Ciencias Sociales en el Centro Universitario del Sur de la Universidad de Guadalajara. Ha realizado diferentes publicaciones, entre ellas: Procuración e impartición de justicia y seguridad ciudadana en el Sur de Jalisco: propuestas ciudadanas y El Emprendurismo en Zapotlán el Grande y su impacto en la zona sur de Jalisco. Correo electrónico: eva.lozano@cusur.udg.mx

Alicia Edith Hermosillo de la Torre. Doctora en Psicología, ha coordinado proyectos de investigación en problemática suicida, prevención y la transferencia de tratamientos empíricamente apoyados con 
financiamientos externos. Destaca el desarrollo y evaluación del programa para el aprendizaje de habilidades socioemocionales PAHSE para la prevención del suicido durante la pre-adolescencia mediante el aprendizaje de habilidades básicas de regulación emocional. Colabora en una red internacional para la prevención de suicidio en estudiantes universitarios PUERTAS, como parte de la iniciativa de encuestas mundiales. Correo electrónico: alicia.hermosillo@edu.uaa.mx 